\title{
Nonlinear Identification of Electronic Brake Pedal Behavior Using Hybrid GMDH and Genetic Algorithm in Brake-By-Wire System
}

\author{
Junhyung Bae*, Seonghun Lee**, Dong-Hwan Shin**, Jaeseung Hong**, Jaeseong Lee** \\ and Jong-Hae Kim ${ }^{\dagger}$
}

\begin{abstract}
In this paper, we represent a nonlinear identification of electronic brake pedal behavior in the brake-by-wire (BBW) system based on hybrid group method of data handling (GMDH) and genetic algorithm (GA). A GMDH is a kind of multi-layer network with a structure that is determined through training and which can express nonlinear dynamics as a mathematical model. The GA is used in the GMDH, enabling each neuron to search for its optimal set of connections with the preceding layer. The results obtained with this hybrid approach were compared with different nonlinear system identification methods. The experimental results showed that the hybrid approach performs better than the other methods in terms of root mean square error (RMSE) and correlation coefficients. The hybrid GMDH/GA approach was effective for modeling and predicting the brake pedal system under random braking conditions.
\end{abstract}

Keywords: Brake-by-wire, Group method of data handling, Genetic algorithm, System identification

\section{Introduction}

The X-by-wire system is a relatively new technology being increasingly implemented in modern automobiles. The X-by-wire system is an automotive system that interprets the driver's inputs and executes appropriate commands to produce desired vehicle behavior, typically via a microprocessor based control system. For fault tolerance, a typical X-by-wire system consists of redundant sensors, actuators, microprocessors, and communication channels. In the X-by-wire equipped vehicle, there are no mechanical or hydraulic connections between the driver's input interface (e.g., throttle, brake, steering) and the target vehicle system $[1,2]$.

The brake-by-wire (BBW) system, a type of X-by-wire, was first introduced in the Mercedes Benz SL series in 2001 [3]. However, that BBW system was decommissioned and removed from the vehicle a few years later due to a number of field problems. Work on the electro-mechanical brake (EMB) in the BBW was being pursued in the late 1990 's by a number of automotive companies, including Bosch, Continental, and TRW [4]. However, issues related to reliability still remain and must be addressed before these systems can be used in an automobile [5-7].

To ensure the BBW system reliability, accurate modeling of the real system is an important step. Models can be used for the EMB analysis, to gain a better understanding of the

$\dagger \quad$ Corresponding Author: Dept. of Electronic and Electrical Engineering, Catholic University of Daegu, Korea. (kjhassk@cu.ac.kr)

* Dept. of Information and Communication Engineering, DGIST, Korea. (baejunh@dgist.ac.kr)

** Convergence Research Center for Future Automotive Technology, DGIST, Korea. ( \{shunlee, sdh77, psman2, jaeseonglee\}@dgist.ac.kr)

Received: May 26, 2016 ; Accepted: February 16, 2017 system. Models also allow us to predict and simulate the system's behaviors. Furthermore, models are necessary for designing new processes, analyzing existing processes, designing controllers, optimizations, supervision, and fault detection and diagnosis. For these purposes, various system identifications have been applied in many fields to model and predict the behavior of complex nonlinear systems based on given input/output data.

Many soft computing approaches for identifying and modeling the nonlinear systems have been proposed. Most of these methods require large amounts of data to estimate the parameters of the model in higher order systems [8]. For example, neural networks and fuzzy logics are used to identify and predict the nonlinear systems based on empirical raw data. Especially, the neural networks are one of the most powerful methods that have been widely employed in recent years in various fields $[9,10]$. However, when using such methods, the nonlinear dynamics are not explicitly expressed as a mathematical model. Therefore, a group method of data handling (GMDH) was first developed by A. G. Ivakhnenko as a multivariate analysis method for complex systems modeling and identification [11]. The main idea in GMDH is to build an analytical function in a feed-forward network based on a quadratic node transfer function whose coefficients are obtained using regression technique. Note that once the analytical GMDH model has been found, application of this model is very quick and cheap. In addition, the GMDH identification is often "black boxes," and they are especially valuable when the underlying physics is complex or uncertain while there is plenty of data to develop a virtual sensor [12]. Consequently, GMDH provides an effective approach for the identification of higher order nonlinear 
systems [13]. However, the GMDH needs a suitable optimization method to find the best network architecture. For the best network architecture, the genetic algorithm (GA) is arranged in a new approach, to design the whole architecture of the GMDH. This provides the optimal number of neurons in each hidden layer and the connectivity configuration needed to find the optimal set of appropriate coefficients of quadratic expressions to model the system [14].

In this paper, first of all, the BBW system was developed. Based on that system, brake pedal sensor signals were measured. Secondly, a hybrid GMDH/GA was applied to identify the electronic brake pedal system for status monitoring. We then evaluated the performance of the hybrid GMDH/GA as a system identification tool.

This paper is organized as follows: Section 2 presents the developed BBW system; Section 3 describes the hybrid GMDH/GA algorithm; applications of the identification for predicting sensor output under random braking conditions are presented in Section 4; the results for identifying the pedal system using different methods are compared in Section 5. Finally, conclusions are presented in Section 6.

\section{Configuration of Developed Brake-By-Wire System}

The developed BBW system architecture is proposed and shown in Fig. 1. It consists of the EMB modules, the electronic brake pedal system, a communication network, main and local electronic control units (ECUs) and power supplies. The EMB actuators are based on the electric motors and a mechanism that applies force to friction pads. The detailed BBW systems are mainly comprised of four types of elements:

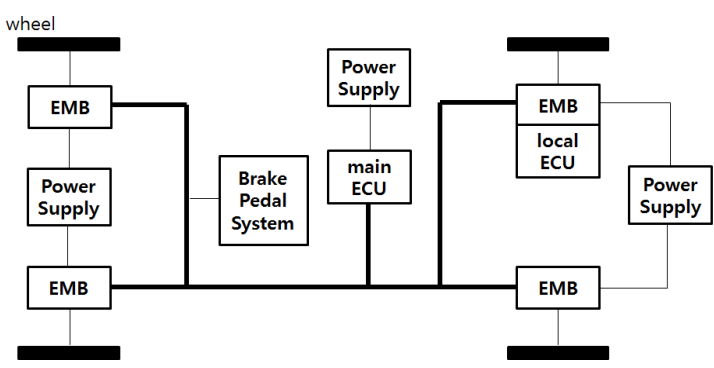

Fig. 1. Architecture of developed BBW system
1. Electronic Control Units (ECUs)

2. Sensors - pedal sensor, hall sensor and encoder

3. Actuator - electric motor, planetary gear, ball screw and caliper

4. Communication network - CAN or FlexRay

Fig. 2 illustrates the block diagram of the developed BBW system. Once the driver inputs a brake command to the system via the brake pedal, a command clamping force signal is generated by the main ECU. This command signal is sent to the EMB local ECU and display via a communication network. The controller uses the command clamping force as a reference input. The controller provides drive control commands for a motor drive module. This module controls three phase currents for the brake actuator which is a permanent magnet synchronous motor (PMSM), energized by a 42 [V] power source. In addition to tracking its reference, the controller also controls the current and position of the PMSM.

\section{Application of Hybrid GMDH/GA to the Electronic Brake Pedal System}

In this section, we describe for a hybrid GMDH/GA algorithm and propose the schematic of brake pedal system identification.

\subsection{GMDH}

The general connection between the inputs and output variables can be expressed by a complicated polynomial series in the form of the Volterra series, known as the Kolmogorov-Gabor polynomial [13]:

$$
y=a_{0}+\sum_{i=1}^{m} a_{i} x_{i}+\sum_{i=1}^{m} \sum_{j=1}^{m} a_{i j} x_{i} x_{j}+\sum_{i=1}^{m} \sum_{j=1}^{m} \sum_{k=1}^{m} a_{i j k} x_{i} x_{j} x_{k}+\cdots
$$

where $x$ is the input to the system, $m$ is the number of inputs and $a$ are coefficients. For most applications the quadratic forms for only two variables, which are called partial descriptions, are used in the form to predict the output as follows.

$$
y=a_{0}+a_{1} x_{i}+a_{2} x_{j}+a_{3} x_{i} x_{j}+a_{4} x_{i}^{2}+a_{5} x_{j}^{2}
$$

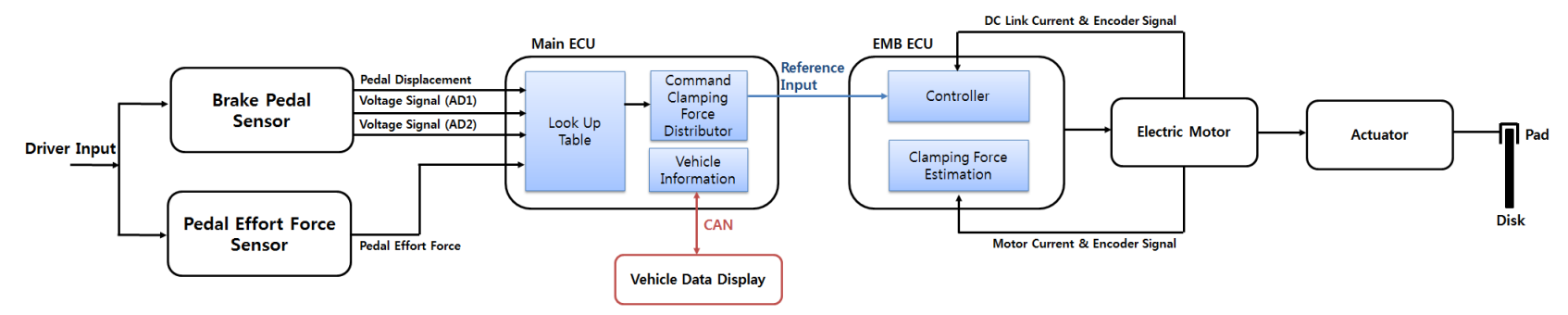

Fig. 2. Block diagram of developed BBW system 
To obtain the value of the coefficients $a$ for each model, a system of Gauss normal equations is solved. The coefficient of nodes in each layer is expressed as follows.

$$
A=\left(X^{T} X\right)^{-1} X^{T} Y
$$

where

$$
\begin{aligned}
Y= & {\left[y_{1}, y_{2}, \ldots, y_{m}\right]^{T}, } \\
A= & {\left[a_{0}, a_{1}, a_{2}, a_{3}, a_{4}, a_{5}\right], } \\
X= & {\left[\begin{array}{cccccc}
1 & x_{1 p} & x_{1 q} & x_{1 p} x_{1 q} & x_{1 p}^{2} & x_{1 q}^{2} \\
1 & x_{2 p} & x_{2 q} & x_{2 p} x_{2 q} & x_{2 p}^{2} & x_{2 q}^{2} \\
\vdots & \vdots & \vdots & \vdots & \vdots & \vdots \\
1 & x_{m p} & x_{m q} & x_{m p} x_{m q} & x_{m p}^{2} & x_{m q}^{2}
\end{array}\right] . }
\end{aligned}
$$

The main function of GMDH is based on the forward propagation of a signal through nodes of the net similar to the principal used in classical neural nets. Every layer consists of simple nodes each of which performs its own polynomial transfer function and passes its output to nodes in the next layer. The basic steps involved in the GMDH modeling are as follows $[15,16]$ :

Step 1: Select input variables $X=\left\{x_{1}, x_{2}, \ldots, x_{m}\right\}$. Divide the available data into training and checking data sets. Before applying the algorithm, the inputs and output are normalized.

Step 2: Construct $k=\left(\begin{array}{c}m \\ 2\end{array}\right)=m(m-1) / 2$ new variables $Z=\left\{z_{1}, z_{2}, \ldots, z_{k}\right\}$ in the training data set and construct the regression polynomial for the first layer by forming the quadratic expression which approximates the output $y$.

Step 3: Identify the contributing nodes at each hidden layer according to the value of the root mean square error (RMSE). Eliminate the least effective variable by replacing the columns of $X$ (old data) by the new columns of $Z$.

Step 4: The GMDH algorithm is carried out by repeating Steps 2 and 3 of the algorithm. When the errors of the checking data in each layer stop decreasing, the iterative computation is terminated.

The network constructed using the self-organizing GMDH algorithm is shown in Fig. 3 [17].

\subsection{Genome representation}

The GA is commonly used in the training of neural networks in terms of associated weights or coefficients, and has successfully performed better than traditional gradient-based techniques. In most GMDH algorithms, the neurons in each layer are only connected to neurons in the adjacent layer. Taking advantage of this feature, it is possible to present a simple encoding scheme for the genotype of each individual in the population $[18,19]$.

In Fig. 4, neurons in the first hidden layer are connected to the output layer by directly going through the second

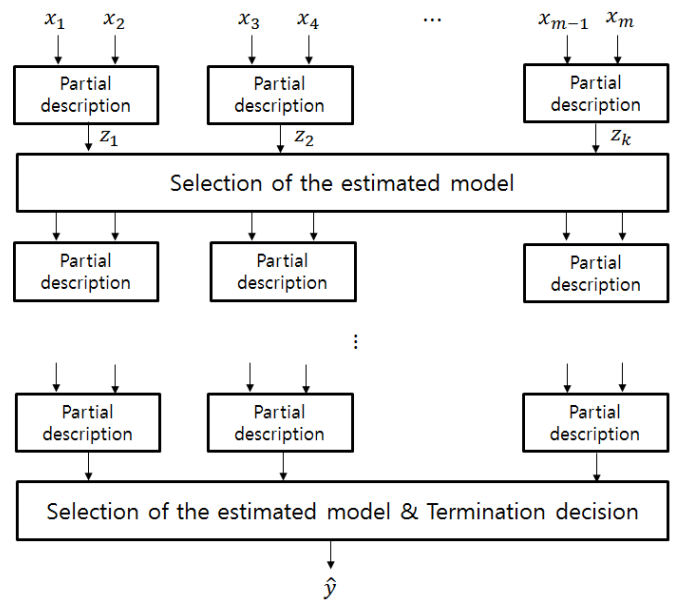

Fig. 3. Self-organizing GMDH algorithm

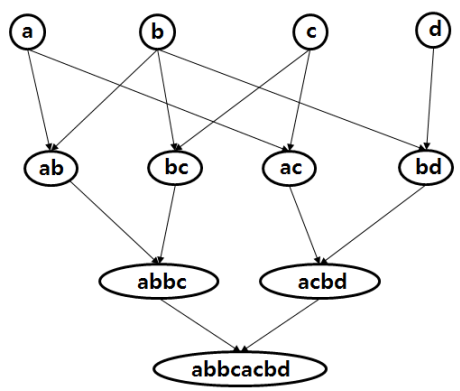

Fig. 4. Genome representation

hidden layer. The genome or chromosome representation, which shows the topology of a GMDH, simply consists of a symbolic string composed of an alphabetic representation of the input variables [20]. In this encoding scheme, each input variable is assigned an alphabetic name, and a chromosome is a string of concatenated substrings of these alphabetic names of inputs. It should be noted that such repetition occurs whenever a neuron passes through some adjacent hidden layers and connects to another neurons in the next $2^{\text {nd }}, 3^{\text {rd }}, \ldots$ following hidden layer. In this encoding scheme, the number of repetitions of that neuron depends on the number of passed hidden layers $l$, and is calculated as $2^{l}$.

\subsection{Proposed scheme of electronic brake pedal system identification}

In the BBW equipped vehicle, the brake pedal sensor is safety-critical component, and this failure will disrupt the vehicle's operation and endanger human lives [21]. The main ECU must always be informed of the driver's intentions to brake or to stop the vehicle, and fault data from the pedal sensor is a serious problem for vehicle control systems. For this reason, the design of a these vehicles need to include safeguards that prevent abnormal behavior. General solutions to this problem include providing hardware redundant sensors, and applying a fail- 


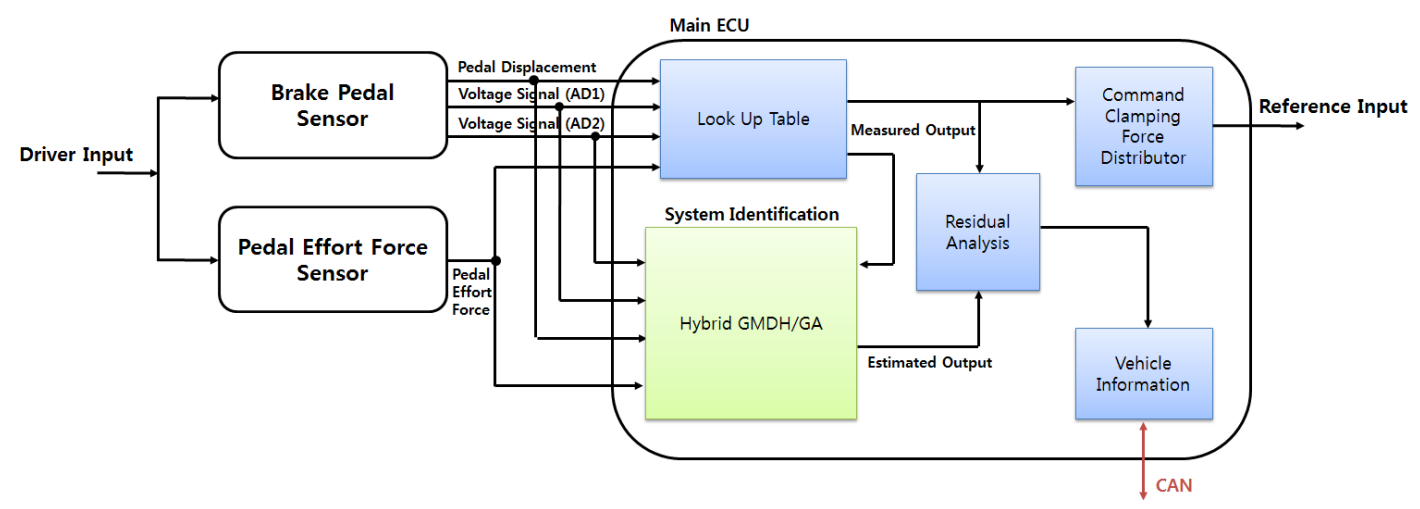

Fig. 5. Proposed scheme of electronic brake pedal system identification using hybrid GMDH/GA

safe mechanism. In addition to the complete loss of sensor information, the ECU may also suffer from an intermittent data loss. This may result from an instantaneous short circuit or disconnection, a communication network fault, or a sudden increase in noise. To address these problems, we introduce a system identification approach based hybrid GMDH/GA as the virtual sensor. The following Fig. 5 shows the proposed scheme of the electronic pedal system identification. In order to model and identify the brake pedal system, the following sensory information is captured as inputs: pedal effort force [kgf], voltage signal AD1 [V], voltage signal AD2 [V] and pedal displacement [\%]. The output is the clamping force command $[\mathrm{N}]$. The residual analysis will conduct the fault diagnosis function; in other words, it will be used with the measured data given by the pedal behavior to compare with the results obtained using the estimated data generated by the hybrid GMDH/GA.

\section{Experimental Results}

\subsection{Experimental setup}

For the system identification, tests were made on a laboratory BBW system. Our BBW system is composed of brake pedal, pedal effort force sensor, ECUs, wheel speed sensors, and EMBs as Figs. 6 and 7. In the EMB system, the electric motor is of the PMSM type with ratings of 250 [W] and 11,000 [rpm] and ensures that the required clamping forces (up to $33[\mathrm{kN}]$ ) can be achieved.

The parameters of interest in this four-input one-output system, which both affect the performance of the clamping force command are the pedal effort force [kgf], voltage signals(AD1[V] and $\mathrm{AD} 2[\mathrm{~V}])$ and pedal displacement [\%]. Accordingly, there were a total of 100,000 experimental data considering four input parameters, as shown in Fig. 8. Out of which 70,000 data points are randomly selected for the training data set while the remaining 30,000 data points are selected for the checking data set. We choose the sampling interval of 0.2 [ms].

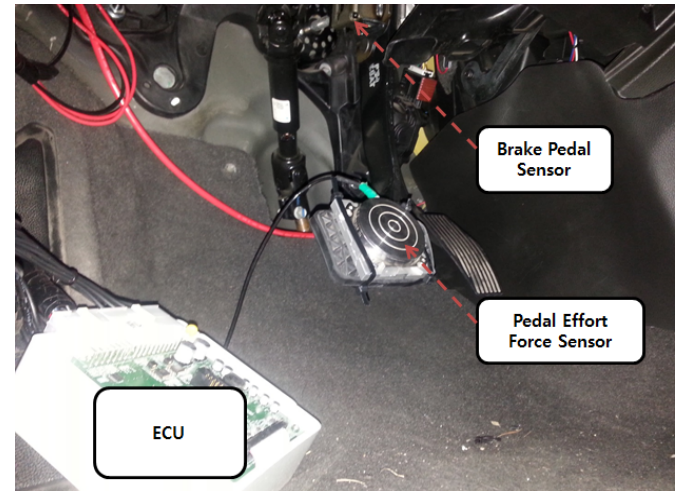

Fig. 6. Electronic brake pedal system

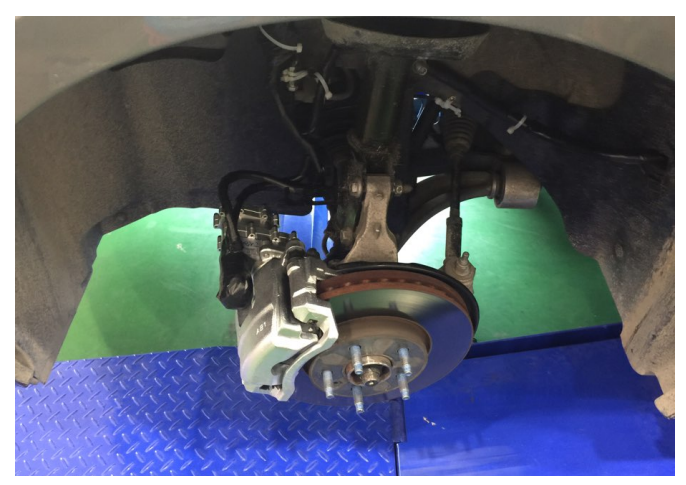

Fig. 7. EMB equipped wheel

In the hybrid GMDH/GA, a population size of 20 was employed together with a crossover probability of 0.9 and a mutation probability of 0.1 in a generation number of 6 and number of hidden layer of 4 . The hybrid GMDH/GA was implemented in MATLAB on a PC with an Intel Core CPU $1.5 \mathrm{GHz}$ and RAM $8 \mathrm{GHz}$. We can construct the structure of the network, which is shown in Fig. 9.

\subsection{Comparison of results and discussions}

The well-known criterion for comparing the different identification methods is the root mean square error (RMSE) and correlation coefficients $\mathrm{R}$ of the predicted 

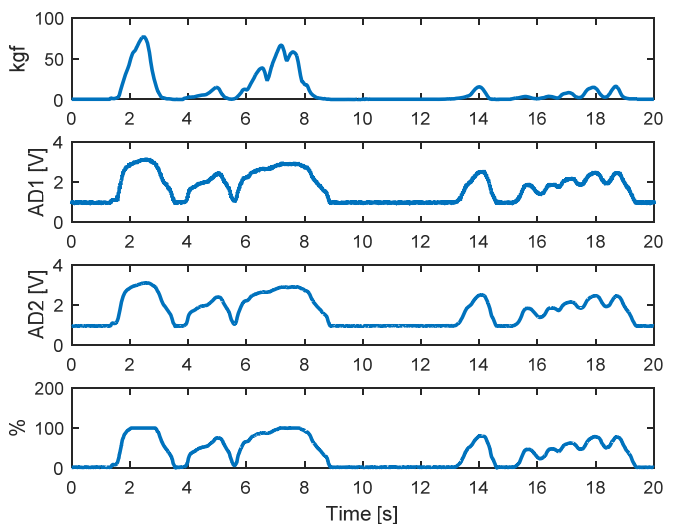

Fig. 8. Experimental data; pedal effort force [kgf] (first), voltage signal AD1 [V] (second), voltage signal AD2 [V] (third) and pedal displacement [\%] (fourth)

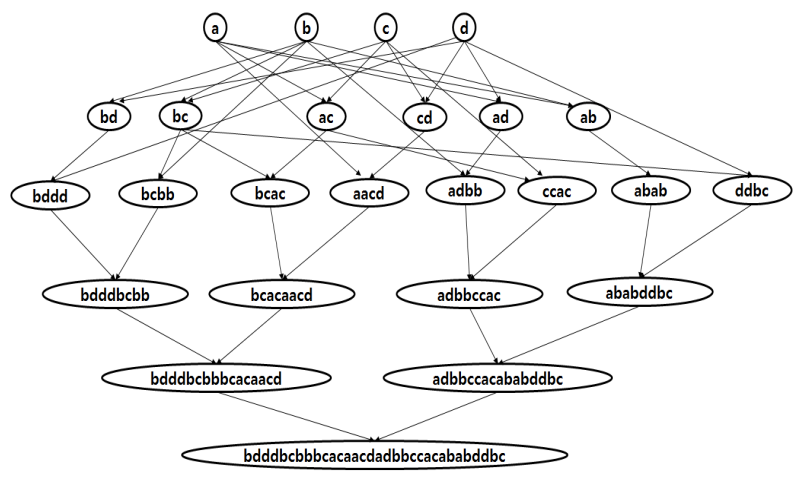

Fig. 9. Network structure of the brake pedal behavior using hybrid GMDH/GA
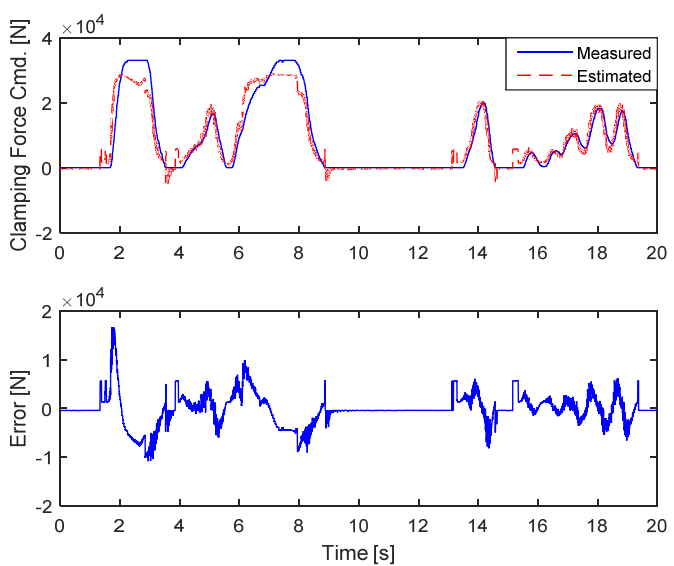

Fig. 10. Neural network identification results

outputs from each model. The reason for using these criterions is that we need to measure the quality of the models and also the predictability of the models.

The neural network was trained for 1000 epochs using the Levenberg-Marquart back-propagation algorithm with a learning rate of 0.001 and an error goal of 0.01 . Figs. 1012 shows the results for the measured, estimated output
Table 1. Comparison of performance results for different identification methods

\begin{tabular}{c|c|c}
\hline Identification & RMSE [N] & $\mathrm{R}$ \\
\hline Neural Network & 3211.8 & 0.95556 \\
\hline GMDH & 1561.8 & 0.98949 \\
\hline Hybrid GMDH/GA & 117.5 & 0.99223 \\
\hline
\end{tabular}
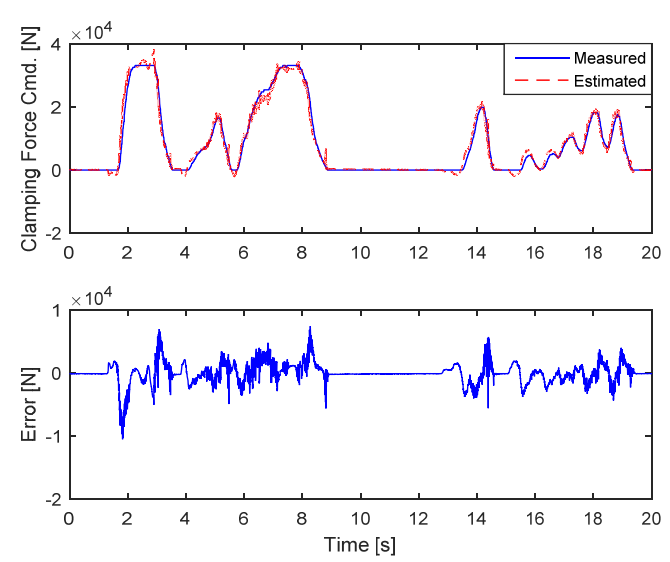

Fig. 11. GMDH identification results
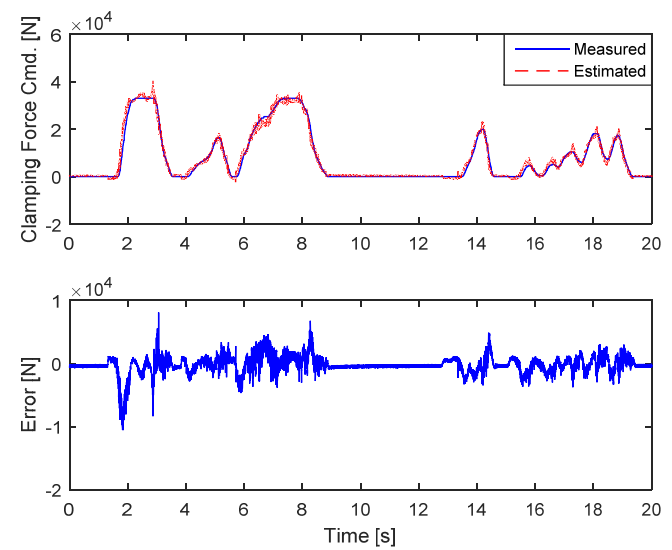

Fig. 12. Hybrid GMDH/GA identification results

data and residual errors for neural networks, GMDH and hybrid GMDH/GA identification, respectively. When a random stepwise and sinusoidal clamping force command is applied, the estimated values follow the target values, which show close agreement with the measured values. Especially, the figures show that proposed hybrid GMDH/ GA identification offers good estimation performance.

Table 1 shows a numerical comparison of the RMSE and $\mathrm{R}$ results from different modeling methods applied to identification of the brake pedal behavior based on Figs. 10-12. As indicated in Table 1, considering the RMSE and $\mathrm{R}$ which are here regarded to be performance indicators, the experimental results clearly demonstrate that the hybrid GMDH/GA outperforms the other identification methods. In the case of the RMSE performance comparison, the hybrid GMDH/GA performed about $92 \%$ better than the GMDH and about $96 \%$ better than the neural network. 
Considering the RMSE and R performance, the hybrid GMDH/GA identification may be the best choice because it had the smallest error compared with the other methods. In addition, the hybrid GMDH/GA identification shows good performance in correlation analysis.

\section{Conclusions}

In this paper, we have presented a hybrid GMDH/GA approach which was successfully used for the modeling and prediction of the brake pedal behavior. In conclusion, the results obtained using the hybrid GMDH/GA were better than those obtained using the other identification methods under random complex braking conditions. The accurate model obtained using the hybrid approach is expected to play an important role in the control and fault diagnosis of the BBW system in the future.

\section{Acknowledgements}

This work was supported by the DGIST R\&D Program of the Ministry of Science, ICT and Future Planning (17IT-01).

\section{References}

[1] S. Anwar, Fault tolerant drive by wire systems: Impact on vehicle safety and reliability, Bentham Science Publishers, 2012.

[2] S. Anwar and L. Chen, "An analytical redundancy based fault detection and isolation algorithm for a road-wheel control subsystem in a steer-by-wire system," IEEE Trans. on Vehicular Technology, vol. 56, no. 5, pp. 2859-2869, Sept. 2007.

[3] A. Higgins and S. Koucky, "Mercedes pumps fly-bywire brakes into new SL roadster," Machine Design, vol. 74, no. 9, p. 26, May 2002.

[4] R. Isermann, R. Schwarz and S. Stölzl, "Fault tolerant drive-by-wire systems," IEEE Control Syst. Magazine, pp. 64-81, Oct. 2002.

[5] Y. Ki, H. Ahn and J. Cheon, "Fault-tolerant control of EMB systems," SAE Int. Journal of Passenger Cars - Electronic and Electrical Systems, vol. 5, no. 2, pp. 579-589, Sept. 2012.

[6] W. Hwang and K. Huh, "Fault detection and estimation for electromechanical brake systems using parity space approach," Journal of Dynamic Systems, Measurement and Control, vol. 137, no. 1, Jan. 2015.

[7] W. Hwang, K. Han, K. Huh, J. Jung and M. Kim, "Model-based sensor fault detection algorithm design for electro-mechanical brake," $4^{\text {th }}$ International IEEE Conf. on Intelligent Transportation Systems, Washington, DC, USA, pp. 962-967, Oct. 2011.
[8] K. Assaleh, T. Shanableh and Y. A. Kheil, "Group method of data handling for modeling magnetorheological dampers," Intelligent Control and Automation, vol. 4, no. 1, pp. 70-79, 2013.

[9] T. López-Molina, A. Pérez-Méndez and F. RivasEcheveria, "Data analysis techniques for neural networks-based virtual sensors," in Proc. of the $8^{\text {th }}$ WSEAS Int. Conf. on Neural Networks, Vancouver, British Columbia, Canada, pp. 76-83, Jun. 2007.

[10] H. Baha and Z. Dibi, "A novel neural network-based technique for smart gas sensors operating in a dynamic environment," Sensors, vol. 9, no. 11, pp. 8944-8960, Nov. 2009.

[11] A. G. Ivakhnenko, "Polynomial theory of complex systems," IEEE Trans. on Systems, Man, and Cybernetics, vol. SMC-1, no. 4, pp. 364-378, Oct. 1971.

[12] D. H. Lim, S. H. Lee and M. G. Na, "Smart softsensing for the feedwater flowrate at PWRs using a GMDH algorithm," IEEE Trans. on Nuclear Science, vol. 57, no. 1, pp.340-347, Feb. 2010.

[13] S. J. Farlow, "The GMDH algorithm of Ivakhnenko," The American Statistician, vol. 35, no. 4, pp. 210-215, Nov. 1981.

[14] M. Shaverdi, S. Fallahi and V. Bashiri, "Prediction of stock price of Iranian petrochemical industry using GMDH-type neural network and genetic algorithm," Applied Mathematical Sciences, vol. 6, no. 7, pp. 319-332, 2012.

[15] J. H. Kim, Y. Matsui, S. Hayakawa, T. Suzuki, S. Okuma and N. Tsuchida, "Acquisition and modeling of driving skills by using three dimensional driving simulator," IEICE Trans. on Fundamentals of Elec., Comm. and Comp., vol. E88-A, no. 3, pp. 770-778, Mar. 2005.

[16] Y. W. Kim, R. Matsuda, T. Narikiyo and J. H. Kim, "Attitude control of planar space robot based on selforganizing data mining algorithm," in Proc. of Int. Conf. on Control, Automation and Syst., Gyeonggi, Korea, pp. 377-382, Jun. 2005.

[17] M. Iwasaki, H. Takei and N. Matsui, "GMDH-based modeling and feedforward compensation for nonlinear friction in table drive systems," IEEE Trans. on Industrial Electronics, vol. 50, no. 6, pp. 1172-1178, Dec. 2003.

[18] N. Nariman-Zadeh, A. Darvizeh, A. Jamali and A. Moeini, "Evolutionary design of generalized polynomial neural networks for modeling and prediction of explosive forming process," Journal of Material Processing and Technology, vol. 164-165, pp. 15161571, May 2005.

[19] N. Nariman-Zadeh and A. Jamali, "Pareto genetic design of GMDH-type neural networks for nonlinear systems," in Proc. of the Int. Workshop on Inductive Modeling, Prague, Czech Republic, pp. 96-103, 2006.

[20] G. C. Onwubolu, Hybrid self-organizing modeling systems, Springer-Verlag Berlin Heidelberg, 2009. 
[21] R. Hoseinnezhad and A. Bab-Hadiashar, "Missing data compensation for safety-critical components in a drive-by-wire system," IEEE Trans. on Vehicular Technology, vol. 54, no. 4, pp. 1304-1311, Jul. 2005.

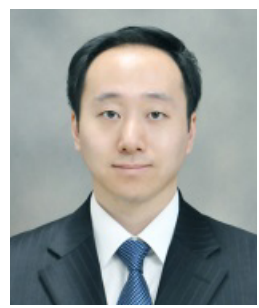

Junhyung Bae He received the B.S degree in electronic engineering and avionics from Korea Aerospace University, Goyang, Korea in 2004 and the M.S. degree in electrical engineering from Hanyang University, Seoul, Korea in 2006. From 2006 to 2010, he was a researcher at the Division of Advanced Industry Science and Technology, Daegu Gyeongbuk Institute of Science and Technology (DGIST), Daegu, Korea. In 2011, he worked as a senior researcher at Samsung Thales, Co., Ltd., Daejeon, Korea. He is currently a researcher at the Convergence Research Center for Future Automotive Technology, DGIST. He is also currently Ph. D. Candidate from the Dept. of Information and Communication Engineering, DGIST. His research interests are state estimation, control and fault diagnosis for an electric vehicles and automotive systems.

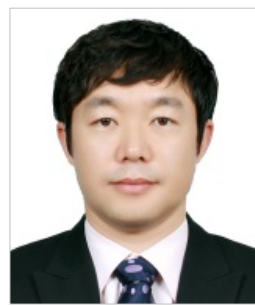

Seonghun Lee He received the B.S., M.S. and Ph.D. degrees in electronic engineering from Kyungpook National University, Daegu, Korea in 1996, 1998 and 2007, respectively. From 1999 to 2002, he was a junior research engineer at Daewoo Precision Co. Ltd., Busan, Korea. Then, from 2002 to 2005, he was a senior research engineer at the Agency for Defense Development (ADD), Daejeon, Korea. He is currently a principal researcher at the Convergence Research Center for Future Automotive Technology, DGIST. His research interests are automotive embedded systems, modeling and simulation for electric vehicles.

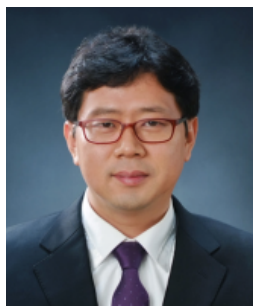

Dong-Hwan Shin He received the B.S., M.S. degree and Ph.D. Candidate in mechanical engineering from Kyungpook National University, Daegu, Korea in 2000, 2002 and 2011, respectively. Since 2006, he has been a senior researcher at the Convergence Research Center for Future Automotive Technology, DGIST, Korea. His research interests are CAE (Computer Aided Engineering) such as multi-body dynamic simulation and structural analysis, electro-mechanical brake, robot end effectors and transfer robots, and spindles with magnetic bearing in machine tool field.

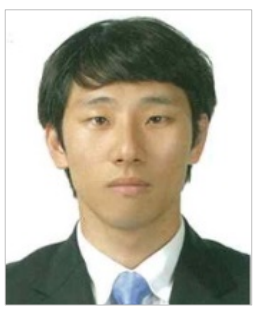

Jaeseung Hong He received the B.S. degree in computer engineering from Dongyang University, Yeongju, Korea in 2008 and the M.S. degree in electrical engineering from Kyungpook National University, Daegu, Korea in 2010. He is currently a research engineer at the Convergence Research Center for Future Automotive Technology, DGIST, Korea. His research interests include the area of real-time embedded system and automotive electronic system.

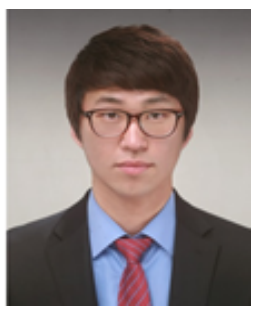

Jaeseong Lee He received the B.S. degree and M.S. degrees in the school of computer and communication engineeering from Daegu University, Gyeongsan, Korea in 2012 and 2015, respectively. He is currently a research engineer at the Convergence Research Center for Future Automotive Technology, DGIST, Korea. His interests include the area of real-time embedded system and automotive electronic system.

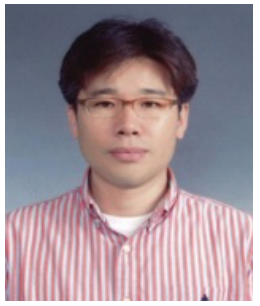

Jong-Hae Kim He received the M.S. and Ph.D. degrees in electrical engineeering from Yeungnam University, Gyeongsan, Korea in 1996 and 1999, respectively. He also received the Ph.D. degree in electrical engineering from Nagoya University, Nagoya, Japan in 2005. From 2005 to 2012, he had been with fundamental technology group of elementary technology team, CDS division, Samsung ElectroMechanics, Co., Ltd., as group leader. Since 2012, he has been an assistant professor with the Dept. of electronic and electrical engineering, Catholic University of Daegu, Gyeongsan, Korea. His research interests are the areas of AC-DC, DC-DC and DC-AC power conversion topology, power factor correction circuit, soft-switching converter topology, wireless power transfer, virtual reality and hybrid/nonlinear control theory. He won the best paper award from ICCAS in 2005. 